\title{
The role of pentraxin 3 in pemphigus vulgaris
}

\author{
Ibrahim Halil Yavuz, Goknur Ozaydın Yavuz \\ Department of Dermatology, Faculty of Medicine, Van Yuzuncu Yil University, Van, Turkey
}

Adv Dermatol Allergol 2020; XXXVII (4): 503-507

DOI: https://doi.org/10.5114/ada.2019.81234

\begin{abstract}
Introduction: Pemphigus is a group of autoimmune bullous diseases caused by antibodies directed against the desmosomal adhesion molecules desmoglein 1 and 3, which are required for intercellular adhesion of keratinocytes. Pentraxins are a group of proteins that function as pattern recognition molecules and also play a role in humoral innate immunity. Pentraxin 3 (PTX3) is the prototype of the long pentraxins and has been shown to be increased in numerous autoimmune diseases.

Aim: To investigate whether PTX3 can be used as a marker of PV caused by autoimmunity and resulting in tissue injury.

Material and methods: The study included 30 patients who presented to the University Medical School Dermatology Department and were diagnosed with PV based on clinical, histological, and immunological findings. The control group included 30 healthy individuals. Human PTX3 concentration was measured with a commercially available ELISA kit, using a double antibody sandwich enzyme-linked immunosorbent assay.

Results: The 60 participants comprised 31 (52\%) men and 29 (48\%) women. The most common site of onset was mucosa + skin $(n=22 ; 73.3 \%)$ and a psychological pathology was present in 7 (23.3\%) patients. Median PTX3 level was significantly higher in the PV group compared to the control group $(p=0.008)$. The ROC curve analysis indicated a significant area under curve (AUC) value for serum PTX3 level in the prediction of PV.
\end{abstract}

Conclusions: PTX3 was found to be increased in PV and PTX3 could be a useful indicator of disease activity in PV.

Key words: autoimmune, pemphigus, pentraxin 3.

\section{Introduction}

Pemphigus is a group of autoimmune bullous diseases caused by antibodies directed against the desmosomal adhesion molecules desmoglein 1 and 3, which are required for intercellular adhesion of keratinocytes [1]. Pemphigus vulgaris (PV) is the most common form of these diseases, with an incidence of 0.1-0.5/100,000 and no gender preponderance. The PV is mostly seen between the fourth and sixth decades of life and is rarely seen in children and the elderly [2]. Although autoimmunity is the underlying cause of PV, the exact etiology of $P V$ remains unknown. Nevertheless, several factors have been blamed, including infections, drugs, environmental factors, tumors, and various human leukocyte antigen alleles [3].

Common clinical manifestations of PV include vesicles and bullae that rupture easily, and a positive Nikolsky's sign is the distinguishing feature of the disease $[2,3]$. Corticosteroids form the primary step in the treatment of PV. However, before the introduction of systemic corticosteroids, most of the patients died within 2 years after diagnosis. With the development of corticosteroids and additional adjuvant therapies over the last decades, a significant reduction in the morbidity and mortality rates has been achieved $[4,5]$.

Pentraxins, also known as pentaxins, are a group of proteins that function as pattern recognition molecules and also play a role in humoral innate immunity. These proteins are divided into two groups: long pentraxins and short pentraxins. C-reactive protein (CRP) and serum amyloid P-component (SAP) are the two typical forms of short pentraxins and pentraxin 3 (PTX3) is the prototype of the long pentraxins. Short pentraxins are produced by hepatocytes while PTX3 is secreted by macrophages, polymorphonuclear cells, neutrophils, endothelial cells, dendritic cells, and smooth muscle cells. Moreover, while short pentraxins are a marker of systemic inflammation, PTX3 reflects local inflammation; therefore, PTX3 has the potential to be a more specific marker for some inflammatory diseases [6, 7]. On the other hand, PTX3 has been shown to be significantly increased in various conditions, particularly including tissue injury and inflammation

Address for correspondence: Ibrahim Halil Yavuz, Department of Dermatology, Faculty of Medicine, Van Yuzuncu Yil University, Van, Turkey, phone: +90 05054753361, e-mail: ihalilyavuz@gmail.com Received: 6.11.2018, accepted: 14.12.2018. 
$[8,9]$. Of note, while the normal serum PTX3 level is $2 \mathrm{ng} / \mathrm{ml}$ in healthy individuals, it can be remarkably high in the setting of inflammation, reaching $800 \mathrm{ng} / \mathrm{ml}$ within 6-8 $\mathrm{h}[7,10]$. In addition, serum PTX3 level has also been shown to be increased in numerous autoimmune diseases [11].

\section{Aim}

In this study, we aimed to investigate whether PTX3 can be used as a marker of PV caused by autoimmunity and resulting in tissue injury.

\section{Material and methods}

The study included 30 patients who presented to the University Medical School Dermatology Department and were diagnosed with PV based on clinical, histological, and immunological findings. The PV was diagnosed according to histopathological criteria including detection of intraepidermal vesicles with acantholysis on hematoxylin staining and IgG and C3 deposition with a fishnet staining pattern within the epidermis directed against cell surface antigens of keratinocytes on direct immunofluorescence staining. Criteria for inclusion in this study: 1) having a diagnosis of pemphigus vulgaris; 2) active blister formation; 3) positive Nikolsky's sign; 4) receiving no treatment; 5) not being in remission. Exclusion criteria included the use of anti-inflammatory drugs that could affect serum PTX3 level within the last 2 weeks and a history of acute and chronic inflammatory diseases including renal and hepatic diseases, cardiac insufficiency, diabetes mellitus, malignancies, pregnancy, psoriatic arthritis, ulcerative colitis, Crohn's disease and PV patients under treatment or in remission The control group included 30 healthy individuals with no family history of autoimmune and bullous diseases and no history of medical treatment. The study was approved by the local ethics committee (Date: 23.03.2018, Number: 33) and a written consent form was obtained from each participant.

\section{Blood sampling and analysis}

Venous blood samples were obtained for routine biochemical analyses in the Dermatology Department. The samples were placed in yellow-top tubes with no anti- coagulant and then transferred to the Biochemistry Department. Following the coagulation process, serum was separated after centrifuging at 1,500 g for 10 min. Human PTX3 concentration was measured with a commercially available ELISA kit, Eastbiopharm (Eastbiopharm, Zhejiang, China), using a double antibody sandwich enzymelinked immunosorbent assay. The PTX3 results were expressed as $\mathrm{ng} / \mathrm{ml}$.

\section{Statistical analysis}

Data were analyzed using SPSS 15.0 (SPSS Inc. Co., Chicago, IL, USA). Normal distribution of data was analyzed using histogram plots and the Kolmogorov-Smirnov test. Descriptive statistics were expressed as mean, standard deviation (SD), and median. Data with non-normal distribution (nonparametric) were compared using the Mann-Whitney U test and Kruskal-Wallis test. Quantitative variables were compared using Spearman's correlation coefficient. A $p$ value of $<0.05$ was considered significant.

\section{Results}

The 60 participants comprised 31 (52\%) men and 29 (48\%) women. No significant difference was found between the two groups with regards to age or gender $(p<0.001)$ (Table 1).

The most common site of onset was mucosa + skin $(n=22 ; 73.3 \%)$ and a psychological pathology (anxiety, depression, bipolar disorder, etc.) was present in 7 (23.3\%) patients (Table 2).

Median PTX3 level was significantly higher in the PV group compared to the control group (2.8 vs. 1.0) $(p=0.008)$ (Table 3)

A ROC curve was produced to determine the cut-off value for PTX3 in the prediction of PV (Figure 1). Based on the ROC curve, the cut-off value was 1.849 and the area under the curve (AUC) was 0.701 (range: 0.547-0.854), which was statistically significant $(p=0.008)$. The sensitivity, specificity, positive predictive value (PPV), and negative predictive value (NPV) for PTX3 were 90\%, 80.1\%, $73.0 \%$, and $87.0 \%$, respectively (Figure 1 ).

The ROC curve analysis indicated a significant AUC value for serum PTX3 level in the prediction of PV. These values are presented in Table 4.

Table 1. Age and gender in both groups

\begin{tabular}{|c|c|c|c|c|c|c|}
\hline \multirow[t]{3}{*}{ Paramete } & & \multicolumn{4}{|c|}{ Group } & \multirow[t]{3}{*}{$P$-value } \\
\hline & & \multicolumn{2}{|c|}{ PV } & \multicolumn{2}{|c|}{ Control } & \\
\hline & & $n$ & $\%$ & $n$ & $\%$ & \\
\hline \multirow[t]{2}{*}{ Gender } & Male & 14 & 46.7 & 15 & 50.0 & \multirow[t]{2}{*}{$0.796^{1}$} \\
\hline & Female & 16 & 53.3 & 15 & 50.0 & \\
\hline Age $^{\star}$ & & $45.7(n=8.7)$ & 44.5 & $46.7(n=8.5)$ & 47.0 & $0.655^{2}$ \\
\hline
\end{tabular}

${ }^{*}$ For quantitative variables, mean $\pm S D$ is used instead of $n$ and median values are used instead of $\% .{ }^{1} \chi^{2}$ test, ${ }^{2}$ independent samples $t$-test. 
Table 2. Clinical characteristics of patients

\begin{tabular}{lccc}
\hline Parameter & & N or mean \pm SD & $\%$ \\
\hline Site of onset & Mucosa + skin & 22 & 73.3 \\
\cline { 2 - 4 } & Mucosa & 6 & 20.0 \\
\cline { 2 - 4 } & Skin & 2 & 6.7 \\
\hline PV-inducing foods? & Yes & 27 & 90.0 \\
\cline { 2 - 4 } & No & 3 & 10.0 \\
\hline Psychological pathology? (anxiety, & Yes & 7 & 23.3 \\
\hline depression, bipolar disorder, etc.) & No & 23 & 76.7 \\
\hline Hospital stay [days] ${ }^{*}$ & & $5.1 \pm 5.3$ & 4.0 \\
\hline Steroids - starting dose $[\mathrm{mg}]^{*}$ (patients who received treatment after being admitted to our clinic) & $77.3 \pm 13.1$ & 80.0 \\
\hline Duration of disease $[y e a r s]^{*}$ & & $5.9 \pm 3.3$ & 5.5 \\
\hline Time to disease control [days] $^{*}$ & & $21.7 \pm 5.3$ & 20.0 \\
\hline
\end{tabular}

${ }^{*}$ For quantitative variables, mean \pm SD is used instead of $n$ and median values are used instead of $\%$.

\section{Discussion}

The results indicated that serum PTX3 level was significantly higher in the PV patients compared to the healthy controls and that PTX3 can be a useful marker for PV. To our knowledge, this is the first study in the literature investigating the relationship between PTX3 and PV.

Autoimmunity in PV has been shown in numerous studies in the literature. In patients with PV, antibodies have mostly been identified in the lesional epidermis and in patients' sera. Moreover, a strong correlation was recently found between serum concentration of antibodies detected by indirect immunofluorescence and the activity and diagnosis of PV [12]. In addition, PV has been shown to be accompanied by various clinical conditions including myasthenia gravis, lupus erythematosus, rheumatoid arthritis (RA), and pernicious anemia [13].

The relationship between PTX3 and autoimmune diseases has been demonstrated in a large body of literature. Assandri et al., for instance, reported that plasma PTX3 level was higher in patients with systemic lupus erythematosus (SLE) compared to healthy controls and the levels decreased after treatment [14]. The study concluded that PTX3 is a significant marker for the diagnosis and follow-up of patients with SLE. Additionally, Sharma et al. revealed that PTX3 was a more sensitive indicator of clinical activity in patients with RA compared to CRP [15]. On the other hand, Huang et al. performed a meta-analysis on 20 studies evaluating the relationship between PTX3 and autoimmune diseases including SLE, rheumatoid arthritis, multiple sclerosis, ankylosing spondylitis, and systemic sclerosis [11]. The analysis showed that serum PTX3 concentration is higher in patients with autoimmune diseases compared to healthy individuals. Similarly, PTX3, together with complement components, has also been shown to have a role in the removal of apoptotic cells in some autoimmune diseases [16, 17]. In our study, we also
Table 3. PTX3 concentration in both groups

\begin{tabular}{lcccc}
\hline Group & \multicolumn{3}{c}{ PTX3 level } & P-value \\
\cline { 2 - 4 } & Mean & \pm SD & Median & \\
\hline PV & 3.1 & 1.5 & 2.8 & 0.008 \\
\cline { 1 - 4 } Control & 3.1 & 4.7 & 1.0 & \\
\hline
\end{tabular}

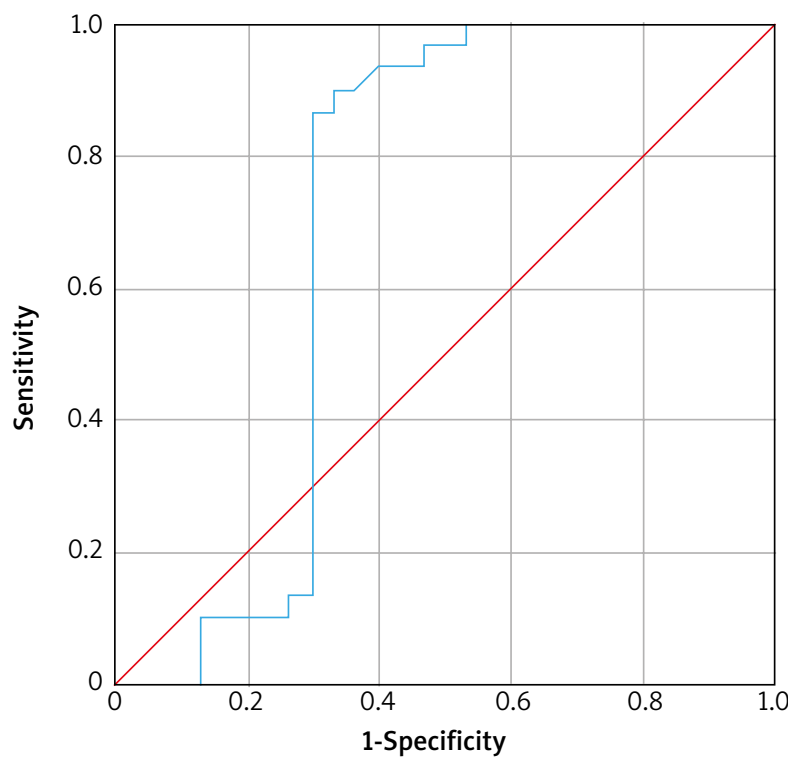

Figure 1. ROC curve for PTX3 in the prediction of PV

Table 4. AUC value for serum PTX3 level in the prediction of PV

\begin{tabular}{ccccc}
\hline & AUC & \multicolumn{2}{c}{$95 \%$ confidence interval $(\mathrm{Cl})$} & \multirow{2}{*}{$\boldsymbol{P}$-value } \\
\cline { 3 - 4 } & value & Lower limit & Upper limit & \\
\hline PTX3 & 0.701 & 0.547 & 0.854 & 0.008 \\
\hline
\end{tabular}


found that serum PTX3 concentration was higher in PV, similarly to other autoimmune diseases.

Literature reviews indicate that there is no clear evidence to date of active bacteria in PV. Nevertheless, it has been shown that the exfoliative toxins of $S$. aureus bind to desmoglein 1 , thereby leading to loss of adhesion, in a similar way to the antibodies in PV [18]. Therefore, some infections are likely to play a role in the activation of PV although they may not be the exact cause of the disease, which implies that the increased PTX3 concentration in PV could be associated with the increased PTX3 concentration in infections. On the other hand, circulating neutrophils migrate to the extravascular sites as a response to tissue injury, thereby leading to the release of PTX3 stored in neutrophils. Subsequently, the resulting inflammation cytokines, particularly IL-1 $\beta$ and TNF- $\alpha$, stimulate PTX3 synthesis during this process $[7,8,19]$.

Increased PTX3 concentration in PV is likely to be caused by the inflammation resulting from acantholysis formation. PTX3 is a key component of humoral immunity, mainly due to its role in the removal of apoptotic cells and cell debris at the site of tissue injury [20]. In a previous meta-analysis, Schmidt and Waschke demonstrated that apoptosis is an important parameter in pemphigus, also noting that nuclear changes and cellular death are the key markers of apoptosis in the setting of acantholysis [17]. In our study, our results also implied that the increased serum PTX3 level in PV could be a result of apoptosis.

It is commonly known that PTX3 does not exist on normal human skin. A previous study showed PTX3 expression in the skin biopsies of patients with severe psoriasis. The study also showed a correlation between PTX3 and the severity and activity of psoriasis [21]. Several other studies have also indicated that PTX3 also has a key role in diseases with a significant dermatological component such as leprosy, urticaria, and Behçet's disease and that it could be a novel marker for these diseases. Moreover, these studies also suggested that PTX3 could be responsible for the expression of inflammatory mediators [22-24]. In our study, PTX3 was found to be increased in PV, as in other inflammatory skin diseases. Based on this finding, it could be asserted that PTX3 could be a more valuable parameter in the activation of PV rather than its diagnosis.

Berksoy Hayta et al. evaluated CRP in 43 patients with PV and 40 healthy volunteers and found that CRP was higher in the PV patients compared to the controls [25]. Similarly, Severo et al. showed that CRP is a useful inflammatory marker in dogs with pemphigus foliaceus. In our study, we evaluated PTX3, which is a similar inflammatory marker to CRP, and we consider that PTX3 is a more specific marker for PV than CRP since PTX 3 is expressed by three different cells and is increased in the setting of local inflammation alone, as compared to CRP, which is produced by the liver alone and is increased in the settings of numerous systemic diseases [26].

Brenner et al. in their study found that garlic compounds led to acantholysis in the in vitro environment. They suggested that foods may have a role in diseases such as pemphigus [27]. Ruocco et al. reported a case of a 49-year-old patient with pemphigus which became worse because of excessive garlic consumption and her disease was controlled following the removal of garlic from the diet [28]. Pietkiewicz et al. evaluated pemphigus patients in the area near a wastewater treatment plant in Poland. The study claimed that some chemical factors (pesticides, thiols, amides, phenols) could trigger autoimmunity in pemphigus [29]. According to a study, the intake of food rich in thiols such as onion, garlic, and leek may be suspected of triggering pemphigus [30]. In our study, we found that most of our patients (90\%) had a high intake of food suspected of triggering pemphigus, which indicates that patients with pemphigus should avoid consuming such foods.

Our study was limited in several ways. First, it was a single-center study with a relatively small patient series. Additionally, no evaluation was performed for other short pentraxins including CRP and SAP and long pentraxins including apexin, neuronal pentraxin 1 (NP1), and neuronal pentraxin 2 (NP2). Accordingly, different outcomes could have been obtained if more pentraxins had been evaluated. PTX3 should not be regarded as a specific marker in PV disease, because PTX3 level can be increased in numerous autoimmune diseases. Therefore, an important drawback of this study is absence of a positive control group. Bullous pemphigoid could be ideal from this point of view, as it is closely related to PV; namely, both diseases share autoimmunity to keratinocyte adhesion molecules.

\section{Conclusions}

PTX3 was found to be increased in PV, as in other autoimmune diseases. Accordingly, PTX3 could be a useful indicator of disease activity in PV. Moreover, compared to the expensive anti-desmoglein antibodies that have been commonly used in the diagnosis and activity of PV over the last decades, PTX3 could be a promising indicator of disease activity due to its reasonable cost. Further prospective studies are needed to substantiate our findings.

\section{Conflict of interest}

The authors declare no conflict of interest.

\section{References}

1. Kasperkiewicz M, Ellebrecht CT, Takahashi H, et al. Pemphigus. Nat Rev Dis Primers 2017; 3: 17026.

2. Kneisel A, Hertl M. Autoimmune bullous skin diseases. Part 2: diagnosis and therapy. J Dtsch Dermatol Ges 2011; 9: 927-47. 
3. Pollmann R, Schmidt T, Eming R, et al. Pemphigus: a comprehensive review on pathogenesis, clinical presentation and novel therapeutic approaches. Clin Rev Allergy Immunol 2018; 54: 1-25.

4. Kridin K. Emerging treatment options for the management of pemphigus vulgaris. Ther Clin Risk Manag 2018; 14: 757-78.

5. Hertl M, Jedlickova H, Karpati S, et al. Pemphigus. S2 Guideline for diagnosis and treatment-guided by the European Dermatology Forum (EDF) in cooperation with the European Academy of Dermatology and Venereology (EADV). J Eur Acad Dermatol Venereol 2015; 29: 405-14.

6. Mantovani A, Garlanda C, Doni A, et al. Pentraxins in innate immunity: from C-reactive protein to the long pentraxin PTX3. J Clin Immunol 2008; 28: 1-13.

7. Cieślik P, Hrycek A. Long pentraxin 3 (PTX3) in the light of its structure, mechanism of action and clinical implications. Autoimmunity 2012; 45: 119-28.

8. Bottazzi B, Inforzato A, Messa M, et al. The pentraxins PTX3 and SAP in innate immunity, regulation of inflammation and tissue remodelling. J Hepatol 2016; 64: 1416-27.

9. Souza DG, Amaral FA, Fagundes CT, et al. The long pentraxin PTX3 is crucial for tissue inflammation after intestinal ischemia and reperfusion in mice. Am J Pathol 2009; 174: 1309-18.

10. Daigo K, Inforzato A, Barajon I, et al. Pentraxins in the activation and regulation of innate immunity. Immunol Rev 2016; 274: 202-17.

11. Huang XL, Zhang L, Duan Y, et al. Association of pentraxin 3 with autoimmune diseases: a systematic review and metaanalysis. Arch Med Res 2016; 47: 223-31.

12. Lanza A, Cirillo N, Femiano F, et al. How does acantholysis occur in pemphigus vulgaris: a critical review. J Cutan Pathol 2006; 33: 401-12.

13. Ruocco E, Wolf R, Ruocco V, et al. Pemphigus: associations and management guidelines: facts and controversies. Clin Dermatol 2013; 31: 382-390.

14. Assandri R, Monari M, Colombo A, et al. Pentraxin 3 plasma levels and disease activity in systemic lupus erythematosus. Autoimmune Dis 2015; 2015: 354014.

15. Sharma A, Khan R, Gupta N, et al. Acute phase reactant, pentraxin 3, as a novel marker for the diagnosis of rheumatoid arthritis. Clin Chim Acta 2018; 480: 65-70.

16. Nauta AJ, Daha MR, van Kooten C, et al. Recognition and clearance of apoptotic cells: a role for complement and pentraxins. Trends Immunol 2003; 24: 148-54.

17. Schmidt E, Waschke J. Apoptosis in pemphigus. Autoimmun Rev 2009; 8: 533-7.

18. Sagi L, Sherer Y, Trau H, et al. Pemphigus and infectious agents. Autoimmun Rev 2008; 8: 33-5.

19. Shiraki A, Kotooka N, Komoda H, et al. Pentraxin-3 regulates the inflammatory activity of macrophages. Biochem Biophys Rep 2016; 5: 290-5.

20. Garlanda C, Bottazzi B, Bastone A, et al. Pentraxins at the crossroads between innate immunity, inflammation, matrix deposition, and female fertility. Annu Rev Immunol 2005; 23: 337-66.

21. Bevelacqua V, Libra M, Mazzarino MC, et al. Long pentraxin 3: a marker of inflammation in untreated psoriatic patients. Int J Mol Med 2006; 18: 415-23.

22. Sağ S, Kamanlı A, Tekeoğlu I, et al. The relationship of pentraxin-3 levels in Behcet disease with IL-17, disease activity and atherosclerotic risk factors. Ann Rheum Dis 2016; 75: 1087-8.
23. Kasperska-Zajac A, Grzanka A, Misiolek M, et al. Pentraxin-3 as a local inflammatory marker in chronic spontaneous urticaria. Cytokine 2015; 76: 566-8.

24. Mendes MA, de Carvalho DS, Amadeu TP, et al. Elevated pentraxin-3 concentrations in patients with leprosy: potential biomarker of erythema nodosum leprosum. J Infect Dis 2017; 216: 1635-43.

25. Berksoy Hayta S, Güner R, Akyol M. Blood mean platelet volume may be predictive for disease course in the cases with pemphigus vulgaris. Biomed Res 2017; 28: 4223.

26. Severo JS, Santana AE, Aoki V, et al. Evaluation of C-reactive protein as an inflammatory marker of pemphigus foliaceus and superficial pyoderma in dogs. Vet Dermatol 2018; 29: 128-e51.

27. Brenner S, Ruocco V, Wolf R, et al. Pemphigus and dietary factors. In vitro acantholysis by allyl compounds of the genus allium. Dermatology 1995; 190: 197-202.

28. Ruocco V, Brenner S, Lombardi ML. A case of diet-related pemphigus. Dermatology 1996; 192: 373-4.

29. Pietkiewicz P, Gornowicz-Porowska J, Bartkiewicz P, et al. Reviewing putative industrial triggering in pemphigus: cluster of pemphigus in the area near the wastewater treatment plant. Adv Dermatol Allergol 2017; 34: 185-91.

30. Ruocco V, Brenner S, Ruocco E. Pemphigus and diet: does a link exist? Int J Dermatol 2001; 40: 161-3. 